\title{
Experimental investigation and modeling of the response surface methodology for the optimization of a multiloop heat pipe
}

Dar-Sun Liou

Department of Navigation, Taipei University of Marine Technology, New Taipei City, Taiwan, darsun8@gmail.com

Follow this and additional works at: https://jmstt.ntou.edu.tw/journal

Part of the Fresh Water Studies Commons, Marine Biology Commons, Ocean Engineering Commons, Oceanography Commons, and the Other Oceanography and Atmospheric Sciences and Meteorology Commons

\section{Recommended Citation}

Liou, Dar-Sun (2021) "Experimental investigation and modeling of the response surface methodology for the optimization of a multiloop heat pipe," Journal of Marine Science and Technology. Vol. 29: Iss. 4, Article 6. DOI: $10.51400 / 2709-6998.1586$

Available at: https://jmstt.ntou.edu.tw/journal/vol29/iss4/6

This Research Article is brought to you for free and open access by Journal of Marine Science and Technology. It has been accepted for inclusion in Journal of Marine Science and Technology by an authorized editor of Journal of Marine Science and Technology. 


\title{
Experimental Investigation and Modeling of the Response Surface Methodology for the Optimization of a Multiloop Heat Pipe
}

\author{
Dar-Sun Liou
}

Department of Navigation, Taipei University of Marine Technology, Tamsui District 25172, New Taipei City, Taiwan

\begin{abstract}
Considering thermohydrodynamic instabilities in the design of cooling systems has become a trend, which has led to the evolution of thermal management. However, such instabilities, which cause flow perturbations, are difficult to explain by using physical theories. The aim of this study was to use a parameter-based modeling technique, namely the response surface methodology (RSM), to characterize the dynamics of multiloop heat pipes (MLHPs) under various heat loads. The RSM, which is based on statistics, was used to determine the relationship between the design parameters and thermal responses of MLHPs, which are represented using polynomials. Th aim of our RSM modeling was to explore the condensation process in MLHPs. Through this exploration, the optimal heating load condition was determined for MLHPs. In the operation range of 10-110 W, MLHPs exhibited high performance at a charge ratio of $31.1 \%-44.2 \%$ and poor performance at a charge ratio from $71 \%$ to $84 \%$. The RSM can be used to find solutions to avoid the failure of chaotic cooling devices.
\end{abstract}

Keywords: Multiloop heat pipe, Response surface methodology, Thermal performance, Thermal management

\section{Introduction}

$\mathrm{M}$ ost industries desire thermal designs in which a large heat load is transferred under a small temperature gradient, and thus, the exploration of thermal management is essential. Such exploration requires the use of high-power mechatronic systems and is difficult. Studies have designed multiloop heat pipes (MLHPs) by considering thermohydrodynamic instabilities $[3,8,17]$. Passive thermal control of MLHPs has attracted considerable research attention, and the heat transfer performance of MLHPs is strongly affected by their thermohydraulic coupling $[9,16,22]$. On the basis of two-phase thermal control of MLHPs, capillary forces can be adjusted to design a closed evaporation-condensation cycle for pumping an unstable working medium.
Many studies have visualized the flow patterns of MLHPs and determined that slug flow is the dominant flow in these pipes [2,20]. Slug flow with bubble oscillation is caused by nucleate boiling. For this flow, the movement of tiny bubbles is regarded as the representative flow pattern in the heating and adiabatic sections $[15,18]$. Thus, the working medium used in low-power MLHP operation is the most critical factor that results in intermittent flow in one direction at high heat loads. To examine the effects of phase changes in the working medium, the heat transfer rate of MLHPs under operating conditions, their cooling method, and their geometric dimensions have been experimentally investigated $[11,21]$. In a previous study, a low-Reynolds-number $k-\epsilon$ turbulence model was developed for assessing the turbulent flow field through two-phase flow modeling. This model indicates the effect of the

Received 28 September 2020; revised 13 January 2021; accepted 4 February 2021.

Available online 3 September 2021.

E-mail address: darsun8@gmail.com. 
Womersley number on oscillating flow. Heat transfer can be a crucial limiting factor for the aforementioned effect. In addition, velocity and temperature characteristics were investigated for various fluid flow and heat transfer conditions in the thermal system [19].

MLHPs are nonequilibrium heat transfer devices in which liquid and vapor slugs are caused by thermohydrodynamic instabilities. Pressure pulsations are induced in MLHPs, and no external source is required to achieve flow oscillation in thermal pipe systems $[6,10,13]$. Most studies on MLHPs have conducted experimental analyses; however, a few studies have investigated the design parameters, especially the interactive effects, of MLHPs. Modeling the individual and interactive effects of MLHPs can enable the optimization of their design parameters.

This study developed a parameter-based modeling technique based on the response surface methodology (RSM) for MLHPs. The RSM has been widely used to model the relationships among the input control variables and output responses of a dynamic system $[4,5,7,12]$. Although the RSM is only an approximation method, a model developed using this methodology can be easily applied even if little information is known about the operational mechanism of a chaotic system [23]. RSM-based modeling has been used in various industrial applications. A model based on experimental temperature responses can be developed for assessing the performance of MLHPs. Such a model can be used to obtain superior MLHP performance under any operating condition.

\section{Thermohydrodynamic instabilities in MLHPs}

In contrast to traditional heat pipes, which have thermal stability, MLHPs are a type of chaotic system with intensive thermal oscillations $[1,14,25]$. In Fig. 1, the local temperature at location A increases when vapor plugs pass this location [Fig. 1(a)]. When the inner wall of the tube is exposed in the vapor or liquid zone, the interaction between the vapor- and liquid-phase media transiently induces flow instability in the tube. In the vapor zone, the vapor-phase medium and the tube wall are separated by a thin liquid film, which is regarded as a fixed interface. This separation results in a high boiling heat transfer coefficient. When evaporation heat transfer occurs, heat energy passes through the thin liquid film. The pressure difference between the two sides of the thin liquid film is expressed as follows: $p_{v}-p_{l}=\frac{2 \sigma}{r}$,

where $\sigma$ and $r$ are the surface tension and curvature radius of the thin liquid film, respectively. At the saturated pressure $P_{v}$, the temperature of vaporphase medium $T_{v}$ is higher than the saturated temperature $T_{\text {sat }}$. Thus, heat energy from the liquid phase is transferred to the vapor phase, which results in the liquid-phase temperature $T_{l}$ being higher than $T_{v}$. Thus, the following equation is obtained:

$T_{l}>T_{v} \geq T_{\text {sat }}$

The inner temperature at location $A$ is expressed as follows:

$T_{A, \text { inner }}=T_{v}+\frac{q}{\alpha_{\text {thin film }}}$

where $q$ is the heat energy passing through the thin liquid film and $\alpha_{\text {thin film }}$ is the heat transfer coefficient when the thin liquid film is evaporated. When the liquid-phase medium enters the heating section, the inner wall is temporarily immersed in the liquid zone [Fig. 1(b)]. At this moment, the local outer surface temperature is expressed as follows:

$T_{A, \text { inner }}=T_{l}+\frac{q}{\alpha_{l}}$

where $\alpha_{1}$ is the heat transfer coefficient of forced liquid convection, which is smaller than $\alpha_{\text {thin film }}$. Thus, the $T_{A, \text { inner }}$ value obtained using Eq. (4) is higher than that obtained using Eq. (3).

When condensation occurs on the thin liquid film, $T_{v}$ is higher than $T_{l}$. Therefore, a reverse heat transfer process occurs at location B [Fig. 1(c) and (d)]. Consequently, $T_{B, \text { inner }}$ can be expressed as follows:

$T_{B, \text { inner }}=T_{v}+\frac{q}{\alpha_{\text {thin film }}}$

The parameter $T_{B, \text { inner }}$ increases when location $A$ is immersed in the vapor-phase medium and decreases when location $A$ is immersed in the liquid-phase medium. The parameter $T_{B, \text { inner }}$ can be expressed as follows:

$T_{B, \text { inner }}=T_{l}-\frac{q_{w, \text { inner }}}{\alpha_{l}}$

The thermal oscillation described in the aforementioned text is the operating mechanism of MLHPs. This oscillation occurs when the inner wall of an MLHP is immersed in the vapor- or liquidphase medium. 


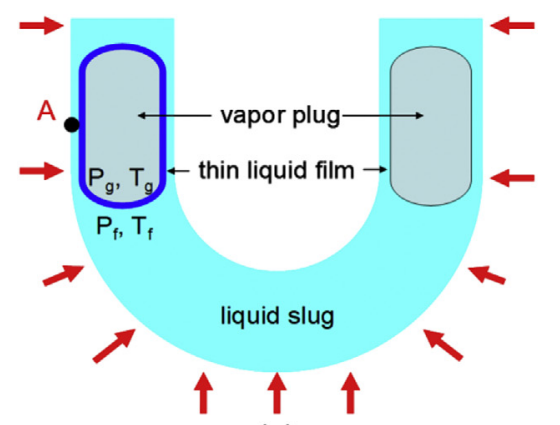

(a)

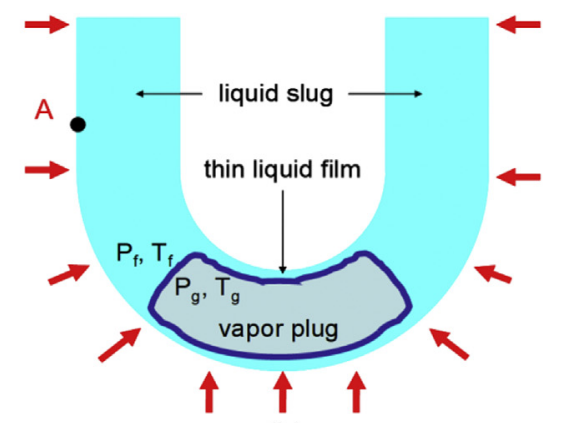

(b)

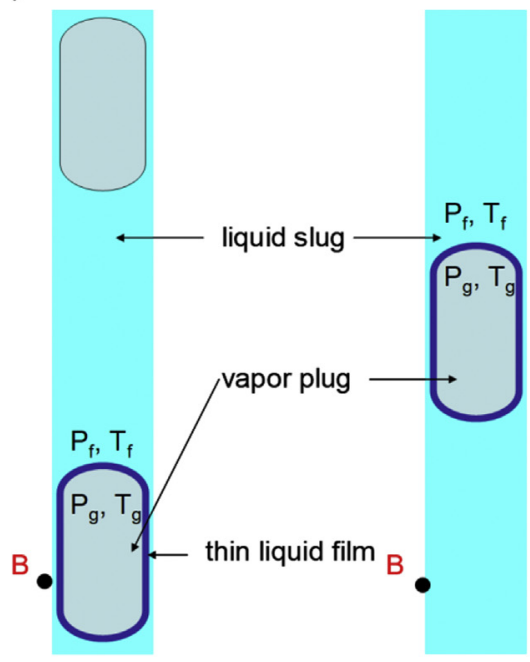

(c)

(d)

Fig. 1. Temperature variation in an MLHP: ( $a$ and $b$ ) heating sections and ( $c$ and $d$ ) cooling sections.

\section{Experimental}

The experimental setup shown in Fig. 2 consists of an MLHP, a data acquisition system, a power supply unit, and thermocouples. The main dimensions of the MLHP were $250 \mathrm{~mm} \times 300 \mathrm{~mm}$, and the pipe was bent using a brass tube with an inner diameter of $2.5 \mathrm{~mm}$. The MLHP comprised a heating section, which had a length of $30 \mathrm{~mm}$, and a cooling section, which was exposed to the environment. Pure water was selected as the working medium due to its large latent heat of evaporation.

The MLHP was vertically operated, and the heat load was provided by a power supply unit (GITEK, model GR-11H12H). Heat was consistently applied to the heating section of the MLHP by using $\mathrm{Ni}-\mathrm{Cr}$ heating wires (NIC80, Omega Engineering Inc., USA) wrapped on the outer side of the brass tube. One capillary tube was selected, and seven calibrated K-type thermocouples (denoted T1-T7; EXGG-K-16, Omega Engineering Inc., USA) were attached to the cooling section of the MLHP at equal intervals. A data acquisition system (DL 750,
YOKOGOWA Inc., Japan) containing thermocouples was used to measure the wall temperatures of the MLHP at a sampling rate of $20 \mathrm{~Hz}$. In each experiment, measurements were conducted for exactly $1 \mathrm{~h}$, and time average results were calculated for the last $10 \mathrm{~min}(12,000$ data points) during normal operation.

To determine the adaptability of the MLHP over a wide operating range, the test charge ratio was varied from $20 \%$ to $90 \%$ and the test heat load was varied from 10 to $110 \mathrm{~W}$. The temperature responses at the measurement locations during the experiment were affected by the tube length, heat load, and charge ratio.

\section{Methodology}

Determining the exact quantitative relationships between the temperature response and design parameters of MLHPs is essential for their analysis. The RSM based on design of experiments (DOE) can be used to obtain the aforementioned relationships in polynomial form as follows: 


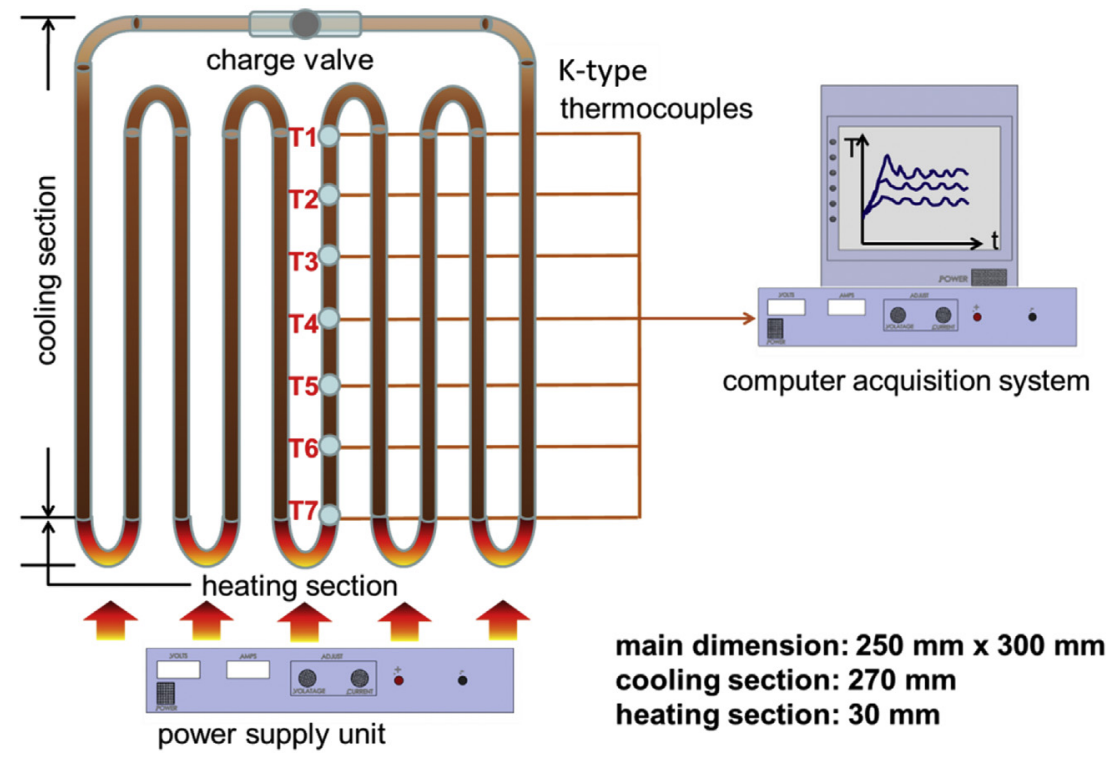

Fig. 2. Schematic of a vertical MLHP system setup for thermal analysis.

$y=F\left(u_{1}, u_{2}, \cdots u_{n}\right)+e$

where $u_{i}$ represents variables, $y$ is the response, $e$ is the error, and $F$ is a combination of variables. A response function can be expressed as follows:

$y=m_{0}+\sum_{i=1}^{n} m_{i} u_{i}+\sum_{i=1, j>i}^{n} m_{i j} u_{i} u_{j}$

where $m$ is a coefficient. When a square form is substituted into Eq. (8), the response function can be represented as follows:

$y=m_{0}+m_{1} u_{1}+m_{2} u_{2}+m_{3} u_{1}^{2}+m_{4} u_{2}^{2}+m_{5} u_{1} u_{2}$

If $u_{3}=u_{1}^{2}, u_{4}=u_{2}^{2}$, and $u_{5}=u_{1} u_{2}$, the following equation is obtained:

$y=m_{0}+m_{1} u_{1}+m_{2} u_{2}+m_{3} u_{3}+m_{4} u_{4}+m_{5} u_{5}$

The coefficient $m_{i}$ can be estimated using a regression model in which the error $(E)$ is considered. The aforementioned regression model can be expressed in vector form as follows:

$Y=U M+E$

where
Table 1. Measured temperature responses along the cooling section of the MLHP.

\begin{tabular}{|c|c|c|c|c|c|c|c|c|}
\hline \multicolumn{9}{|c|}{ Temperature response $\left({ }^{\circ} \mathrm{C}\right)$} \\
\hline \multicolumn{2}{|c|}{$\begin{array}{l}\text { Operational } \\
\text { condition }\end{array}$} & \multirow{2}{*}{$\begin{array}{l}\mathrm{T} 1_{\mathrm{av}} \\
26.3\end{array}$} & & \multirow{2}{*}{$\begin{array}{r}\mathrm{T} 3_{\mathrm{av}} \\
27.3\end{array}$} & & \multirow{2}{*}{$\begin{array}{l}\mathrm{T} 5_{\mathrm{av}} \\
31.6\end{array}$} & \multirow{2}{*}{$\begin{array}{c}\mathrm{T} 6_{\mathrm{av}} \\
38.2\end{array}$} & \multirow{2}{*}{$\begin{array}{l}\mathrm{T} 7_{\mathrm{a}} \\
46.6\end{array}$} \\
\hline $20 \%$ & $10 W$ & & & & & & & \\
\hline & $30 W$ & 26.8 & 27.4 & 28.8 & 32.4 & 40.3 & 55.4 & 71.2 \\
\hline & $50 \mathrm{~W}$ & 28.3 & 29.7 & 35.0 & 50.2 & 75.2 & 82.9 & 85.7 \\
\hline & $70 W$ & 31.8 & 36.2 & 48.2 & 73.7 & 88.9 & 90.2 & 91.2 \\
\hline & $90 W$ & 42.8 & 45.2 & 55.3 & 68.5 & 80.1 & 85.0 & 85.5 \\
\hline & $110 \mathrm{~W}$ & 49.8 & 56.7 & 67.6 & 77.6 & 86.7 & 88.5 & 89.9 \\
\hline \multirow[t]{6}{*}{$40 \%$} & $10 W$ & 26.6 & 26.9 & 27.4 & 29.1 & 32.2 & 38 & 46.6 \\
\hline & $30 W$ & 28.5 & 28. & 30.3 & 37.8 & 50. & 60 & 63.2 \\
\hline & $50 W$ & 32.4 & 34.3 & 34 & 45.7 & 59. & 70 & 73.3 \\
\hline & $70 W$ & 43.3 & 44.0 & 49.5 & 66.8 & 73.9 & 76 & 77.6 \\
\hline & $90 \mathrm{~W}$ & 51.3 & 52 & 66. & 74 & 86. & 91 & 92.8 \\
\hline & $110 \mathrm{~W}$ & 58.2 & 59.2 & 65.6 & 72.3 & 82.4 & 91.5 & 95.0 \\
\hline \multirow[t]{6}{*}{$60 \%$} & $10 W$ & 28.7 & 29.2 & 30.5 & 31.9 & 37.5 & 43.2 & 53.4 \\
\hline & $30 W$ & 32.2 & 32.3 & 36.1 & 40.7 & 52.1 & 62 & 73.4 \\
\hline & $50 W$ & 40.3 & 41.3 & 46.3 & 50.8 & 61.2 & 68 & 78.9 \\
\hline & $70 \mathrm{~V}$ & 40.1 & 42.0 & 45. & 48.8 & 57. & 64 & 76.8 \\
\hline & $90 W$ & 54.3 & 59. & 62. & 65.5 & 75. & 81 & 89.1 \\
\hline & 110 & 56.2 & 62 & 71 & 79 & 87 & 90 & 92.7 \\
\hline \multirow[t]{6}{*}{$80 \%$} & $10 W$ & 25.0 & 25.5 & 26.0 & 27.1 & 31.9 & 39 & 48.5 \\
\hline & $30 W$ & 27.1 & 27.8 & 29.3 & 32.2 & 44.0 & 58.9 & 76.1 \\
\hline & $50 W$ & 34.3 & 35.1 & 37.5 & 39.6 & 52.8 & 64.8 & 79.8 \\
\hline & $70 W$ & 41.6 & 42.1 & 44.0 & 45.2 & 59.5 & 69.5 & 82.1 \\
\hline & $90 W$ & 52.2 & 52.3 & 52.8 & 53.5 & 68.4 & 77.3 & 88.2 \\
\hline & $110 \mathrm{~W}$ & 60.7 & 62.0 & 62.3 & 62.6 & 77.7 & 85.4 & 94.8 \\
\hline \multirow[t]{6}{*}{$90 \%$} & $10 \mathrm{r}$ & 26.9 & 27 & 27.8 & 28.3 & 32.2 & 37.0 & 44.0 \\
\hline & $30 \mathrm{~V}$ & 27.0 & 27.6 & 29.4 & 32.0 & 41.5 & 54.7 & 71.9 \\
\hline & $50 \mathrm{~W}$ & 30.2 & 32.2 & 35.9 & 42.1 & 53.1 & 65.9 & 80.4 \\
\hline & $70 \mathrm{~W}$ & 35.6 & 38.4 & 42.7 & 49.2 & 59.8 & 71.5 & 84.2 \\
\hline & $90 \mathrm{~W}$ & 49.8 & 53.1 & 57.9 & 64.0 & 76.7 & 87.0 & 95.2 \\
\hline & $110 \mathrm{~W}$ & 58.4 & 61.6 & 66.2 & 71.4 & 83.3 & 92.4 & 99.1 \\
\hline
\end{tabular}




$$
\begin{aligned}
Y=\left[\begin{array}{l}
y_{1} \\
y_{2} \\
\vdots \\
y_{n}
\end{array}\right], X & =\left[\begin{array}{llll}
1 & u_{11} & u_{12} & \cdots u_{1 k} \\
1 & u_{21} & u_{22} & \cdots u_{2 k} \\
\vdots & \vdots & \vdots & \ddots \\
1 & u_{n 1} & u_{n 2} & \cdots u_{n k}
\end{array}\right], M \\
& =\left[\begin{array}{l}
m_{1} \\
m_{2} \\
\vdots \\
m_{k}
\end{array}\right], \text { and } E=\left[\begin{array}{l}
e_{1} \\
e_{2} \\
\vdots \\
e_{n}
\end{array}\right],
\end{aligned}
$$

For the coefficient vector $M$, the unbiased estimator $b$ can be determined using the least squares error method as follows:
$b=\left(U^{T} U\right)^{-1} U^{T} Y$,

The covariance matrix of $b$ can be obtained as follows:

$\operatorname{cov}\left(b_{i}, b_{j}\right)=C_{i j}=\sigma^{2}\left(U^{T} U\right)^{-1}$,

where $\sigma^{2}$ is the estimated squared error.

$$
\sigma^{2}=\frac{S S_{E}}{n-k-1},
$$

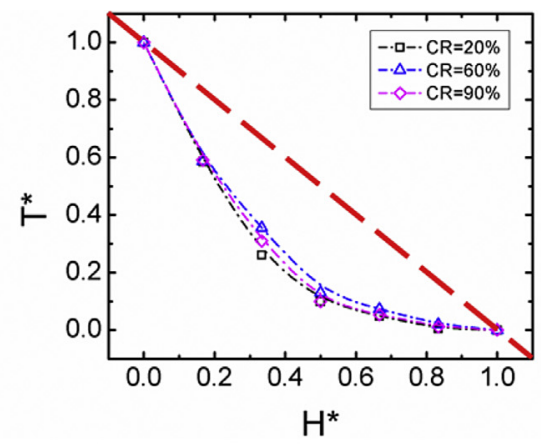

(a)

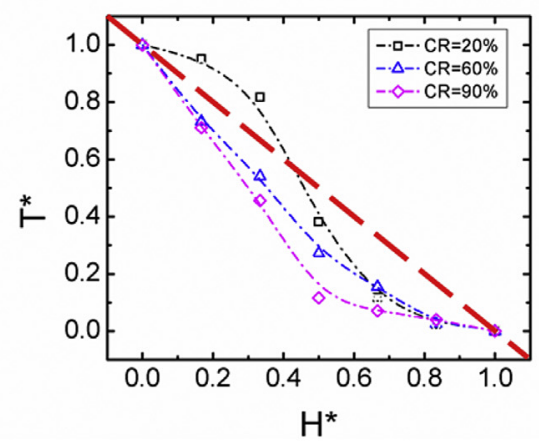

(c)

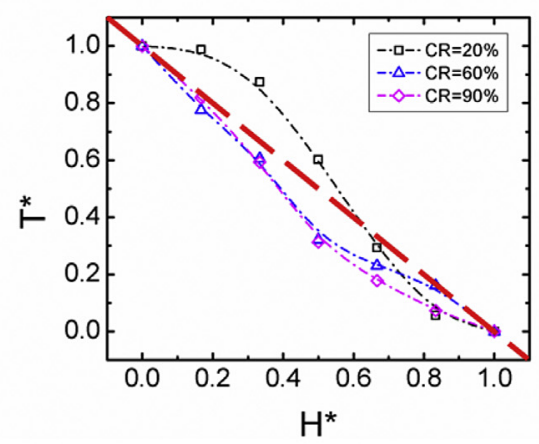

(e)

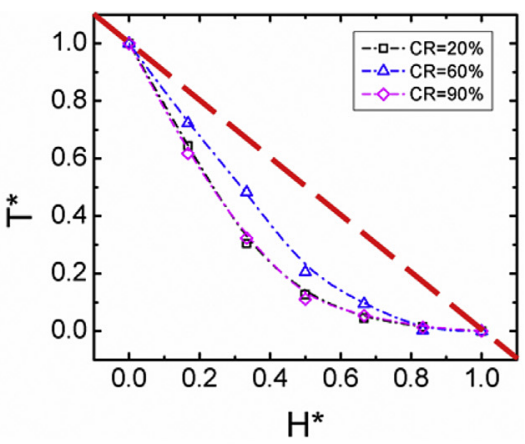

(b)

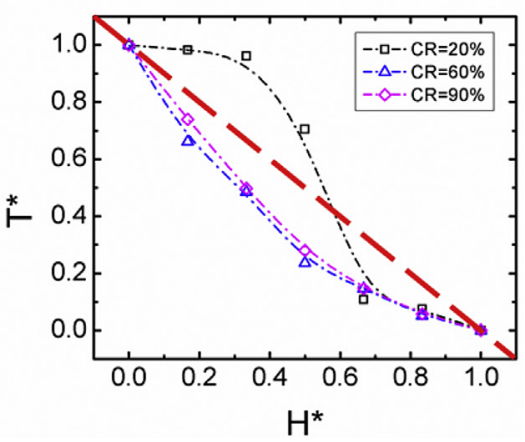

(d)

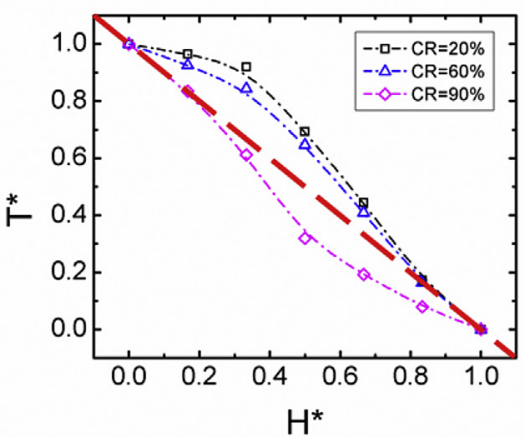

(f)

Fig. 3. Comparison of the normalized temperature distributions along the cooling section for different charge ratios at heat loads of (a) 10, (b) 30, (c) 50, (d) 70, (e) 90, and (f) $110 \mathrm{~W}$. 
where $S S_{E}$ is the sum of the squared residuals and is expressed as follows:

$S S_{E}=Y^{T} Y-b^{T} U^{T} Y$

The performance of the developed RSM models is determined in terms of $R_{a d j}^{2}\left(R^{2}\right.$ adjusted), which is expressed as follows:

$R_{a d j}^{2}=1-\frac{S S_{E} /(n-k-1)}{S_{y y}(n-1)}$,

where $S_{y y}$ is the sum of squares and is calculated as follows:

$S_{y y}=Y^{T} Y-\frac{\left(\sum_{i=1}^{n} y_{i}\right)^{2}}{n}$,

All the coefficients of the response functions can be evaluated using the $t$-statistic, which is represented as follows:

$t_{0}=\frac{b_{j}}{\sqrt{s^{2} C_{j j}}}$,

where $C_{j j}$ is the element of the covariance matrix and $b_{j}$ is a coefficient.

The RSM models developed in this study model the effects of key parameters, such as the charge ratio, heat load, and length of cooling section, on the temperature response of the MLHP. The temperature variations along the cooling section are determined to estimate the heat transfer capacity of the MLHP as follows:

$Q=m C_{p}\left(T 7_{a v}-T 1_{a v}\right)$,
The total thermal resistance of the MLHP is determined as follows:

$R_{t o t a l}=\frac{\left(T 7_{a v}-T 1_{a v}\right)}{Q}$

All the developed RSM models were validated through deviation analysis. Deviation is defined as the relative error between the prediction and measurement results.

\section{Results and discussion}

The thermal performance of MLHPs is affected by their thermohydrodynamic coupling, geometry, and operating conditions [24]. Therefore, the discussion includes information on the geometric and operation parameters.

The mean temperature responses along the cooling section were obtained for various conditions (Table 1). These mean responses were used to investigate the MLHP dynamics. The low, medium, and high charge ratios considered were $20 \%, 60 \%$, and $90 \%$, respectively. The slope of the temperature distribution versus height plot represents an idealized situation for constant heat loss along the cooling section of the MLHP. At a low heat load of $10 \mathrm{~W}$, all the temperature responses exhibited similar decreasing trends [Fig. 3(a)]. The fluid charge ratio had no significant effect on the MLHP under the aforementioned heating condition; thus, the MLHP could be considered stationary.

When the heat load was increased to $30 \mathrm{~W}$, the temperature response for a charge ratio of $60 \%$ was higher than those for the other charge ratios [Fig. 3(b)]. The heat transfer modes began to change

Table 2. Temperature response models developed for the MLHP.

\begin{tabular}{ll}
\hline Complexity & Model structure \\
\hline Square polynomials & $T 1_{a v}=16.00647-0.036294 Q+0.48876 C R+2.32500 e^{-003} Q^{2}$ \\
& $-4.65266 e^{-003} C R^{2}+1.24547 e^{-003} Q^{*} C R$ \\
& $T 2_{a v}=16.52952+2.88490 e^{-003} Q+0.44591 C R+2.45956 e^{-003} Q^{2}$ \\
Cubic polynomials & $-4.15891 e^{-003} C R^{2}+8.81279 e^{-004} Q^{*} C R$ \\
& $T 3_{a v}=16.30792+0.19586 Q+0.38325 C R+2.08214 e^{-003} Q^{2}$ \\
& $-3.32041 e^{-003} C R^{2}-6.37544 e^{-000} Q^{*} C R$ \\
& $T 4_{a v}=-6.74202+0.80014 Q+1.81404 C R+3.27003 e^{-003} Q^{2}$ \\
& $-0.030259 C R^{2}-0.014593 Q^{*} C R-4.20139 e^{-005} Q^{3}$ \\
& $+1.64287 e^{-004} C R^{3}+7.30074 e^{-005} Q^{2 *} C R$ \\
& $+3.54837 e^{-005} Q^{*} C R^{2}$ \\
& $T 5_{a v}=-5.02101+1.85511 Q+1.27540 C R-9.10104 e^{-003} Q^{2}$ \\
& $-0.01701 C R^{2}-0.02318 Q^{*} C R-1.13426 e^{-006} Q^{3}$ \\
& $+8.02368 e^{-005} C R^{3}+1.18794 e^{-004} Q^{2 *} C R$ \\
& $+6.86632 e^{-005} Q^{*} C R^{2}$ \\
& $T 6_{a v}=2.10348+2.22453 Q+1.19820 C R-0.01980 Q^{2}-0.01899 C R^{2}$ \\
& $-0.01777 Q^{*} C R+6.15741 e^{-005} Q^{3}+1.01941 e^{-004} C R^{3}$ \\
& $+8.98220 e^{-005} Q^{2 *} C R+6.08369 e^{-005} Q^{*} C R^{2}$ \\
& $T 7_{a v}=37.22471+2.07940 Q-0.59121 C R-0.024150 Q^{2}$ \\
& $+0.013775 C R^{2}-6.86068 e^{-003} Q^{*} C R+1.00648 e^{-004} Q^{3}$ \\
& $-8.53711 e^{-005} C R^{3}+2.81849 e^{-005} Q^{2 *} C R+3.68715 e^{-005} Q^{*} C R^{2}$
\end{tabular}


under a heat load of $30 \mathrm{~W}$ and a charge ratio of $60 \%$. When the heat load was higher than $50 \mathrm{~W}$, the MLHP was excited under all fluid charge conditions [Fig. 3(c)-(f)]. Thus, the MLHP could be operated at all charge ratios when the heat load was $50 \mathrm{~W}$. For a charge ratio of $20 \%$, small temperature variations were observed when $0<H^{*}<0.5$ and large temperature variations were observed when $0.5<H^{*}<$ 1. At the aforementioned charge ratio, the latent heat transfer in the lower part of the tube was considerably higher than that in the upper part of the tube. The opposite result was obtained at a charge ratio of $90 \%$. At a charge ratio of $60 \%$, the temperature varied relatively evenly along the tube. The slope was a suitable indicator for determining the conditions under which the MLHP exhibited superior performance.

The temperature variations at different locations in the cooling section of the MLHP were measured and modeled. By using RSM models, the temperature responses are expressed as polynomials related to the heat load and charge ratio (Table 2). The parameters $T 1_{a v}$ to $T 3_{a v}$ were fitted with secondorder polynomials, whereas the parameters $T 4_{a v}$ to $T 7_{a v}$, were fitted with third-order polynomials; doing so validated the increased instability of the thermohydrodynamics around the heat load, corresponding to the physical feature of cooling devices. Fig. 4 shows the correlations between the measured and predicted values of $T 1_{a v}, T 4_{a v}$ and $T 7_{a v}$. The predicted results deviated marginally from the experimental results. The deviations for $T 1_{a v}$, $T 4_{a v}$, and $T 7_{a v}$ were -0.134 to $0.084,-0.138$ to 0.157 , and -0.086 to 0.111 , respectively. The polynomial order was not consistent for all the temperature responses, indicating that the tube length affected the thermal performance of the MLHP. The response surfaces and contour plots for $T 1_{a v}, T 4_{a v}$ and $T 7_{a v}$ are displayed in Fig. 5. This figure indicates the effects of the charge ratio and heat load on the temperature responses. A comparison of the aforementioned three contour plots indicates that the charge ratio had major influences on $T 1_{a v}$ and $T 7_{a v}$ at low and high heat loads, respectively. Moreover,

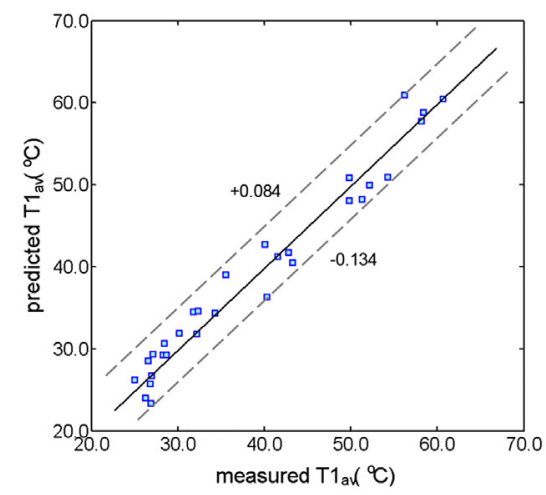

(a)

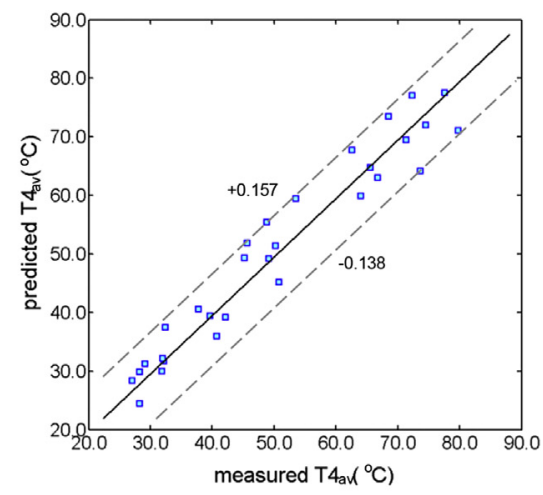

(b)

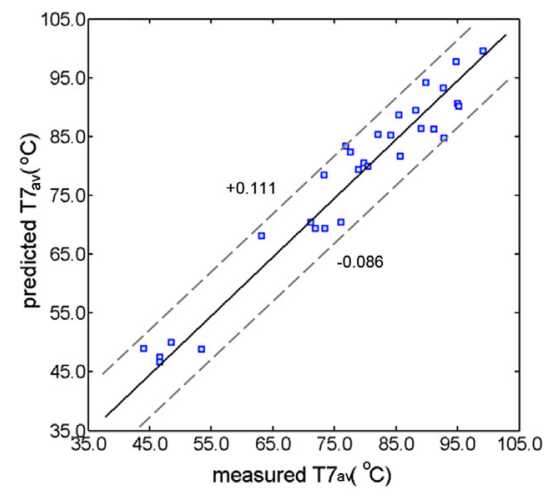

(c)

Fig. 4. Correlations between the measured and predicted values of (a) $T 1_{a v}$ (b) $T 4_{a v}$ and (c) $T 7_{a v}$. 
the charge ratio had the same effect on $T 4_{a v}$ at various heat loads.

The cooling process of the MLHP is assessed according to the consistency of the flow oscillation along the tube. A temperature model can be established for the cooling section of the MLHP on the basis of the length of this section by using the RSM. A normalized temperature response model is suitable for determining the effects of design parameters on general MLHPs. Such a model can be represented as follows:
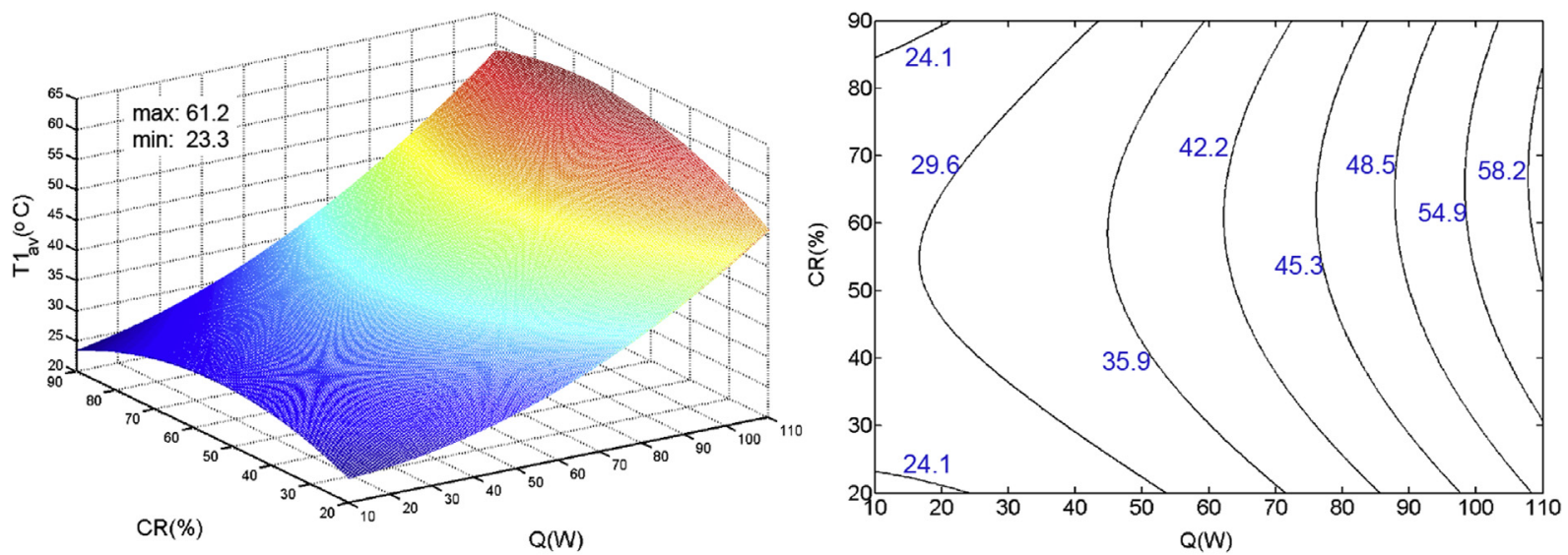

(a)
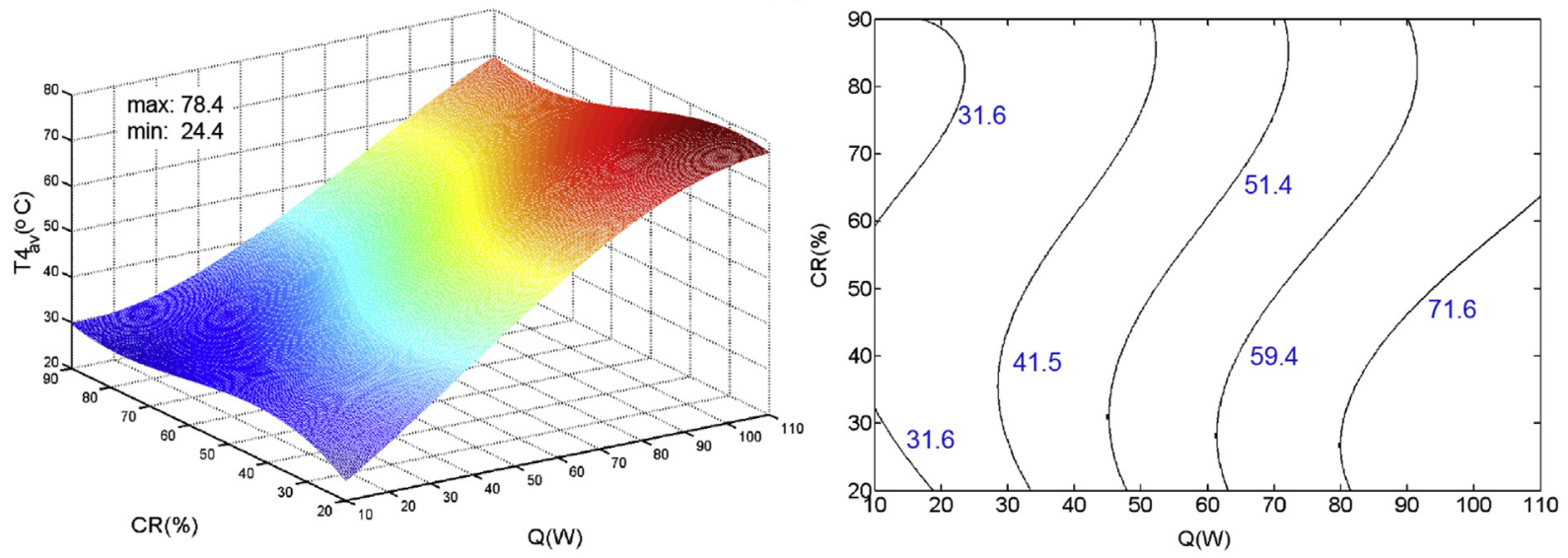

(b)
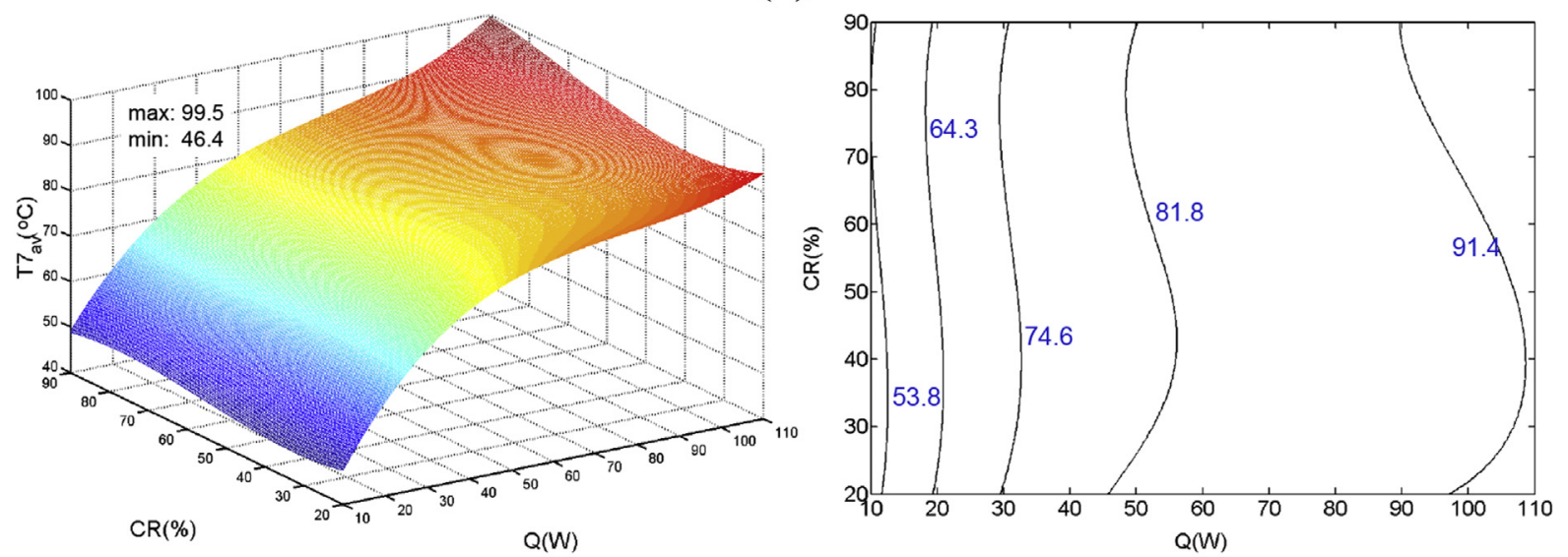

(c)

Fig. 5. Response surfaces and contours obtained for the prediction models of (a) $T 1_{a v}$ (b) $T 4_{a v}$ and (c) $T 7_{a v}$. 


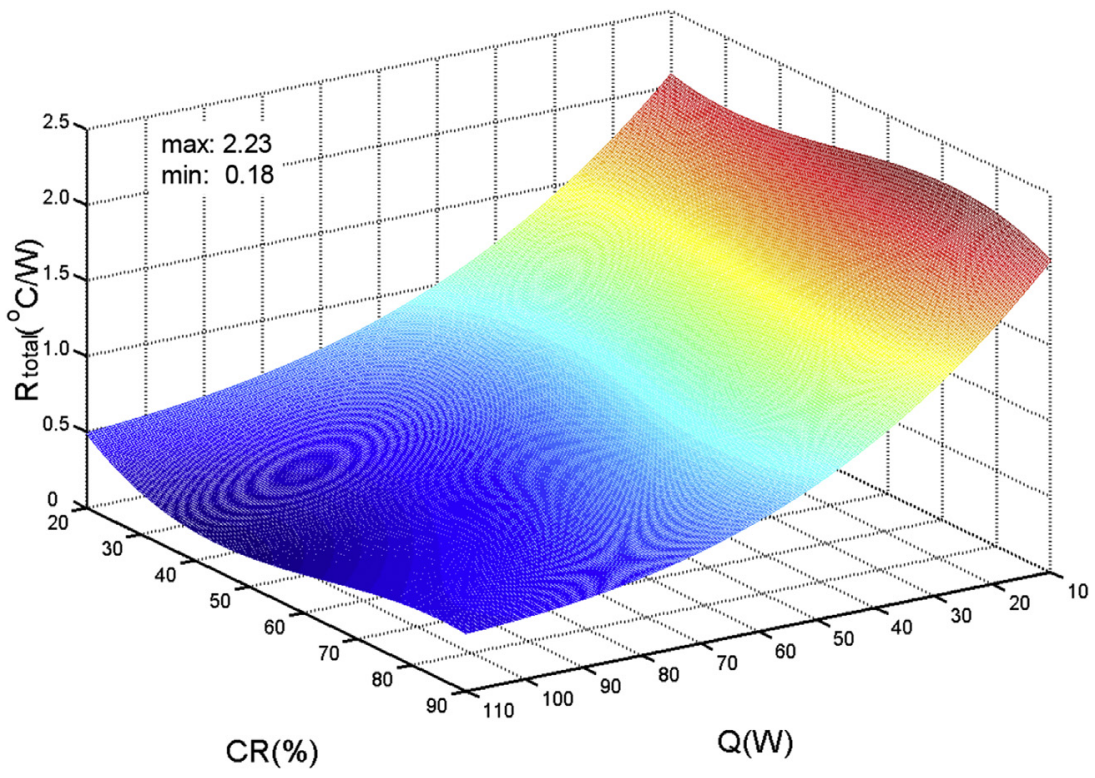

(a)

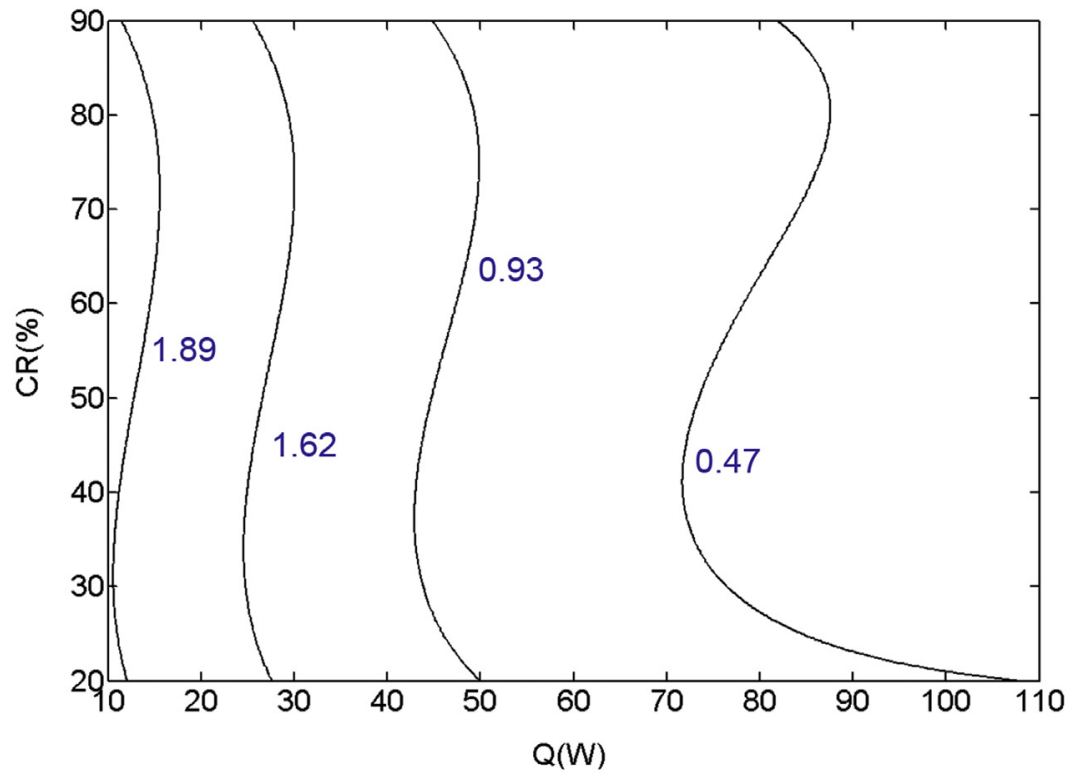

(b)

Fig. 6. Response surface and contours of the $R_{\text {total }}$ model.

$$
\begin{aligned}
T^{*}= & 0.0054+0.1861 Q^{*}+0.6152 C R^{*}-0.1932 H^{*} \\
& +0.1553 Q^{* 2}-0.6526 C R^{* 2}+1.0709 H^{* 2} \\
& -0.5245 Q^{*} * C R^{*}+1.7674 Q^{*} * H^{*} \\
& -1.1031 C R^{*} * H^{*}+0.0421 Q^{* 3}+0.1753 C R^{* 3} \\
& -0.5270 H^{* 3}+0.4157 Q^{* 2} * C R^{*}-1.0193 Q^{* 2} * H^{*} \\
& +0.1367 Q^{*} * C R^{* 2}-0.5106^{*} * H^{* 2} \\
& +0.4728 C R^{* 2} * H^{*}+0.5840 C R^{*} * H^{* 2} \\
& -0.0966 Q^{*} * C R^{*} * H^{*}
\end{aligned}
$$

where the superscript * indicates a normalized parameter. A normalized model for predicting MLHP performance can also be derived on the basis of data in Table 2 and Eq. (21) as follows:

$$
\begin{aligned}
& R_{\text {total }}^{*}=0.9827-1.8842 Q^{*}-0.3666 C R^{*}+1.5637 Q^{* 2} \\
& \quad+1.4108 C R^{* 2}-0.7433 Q^{*} * C R^{*}-0.4595 Q^{* 3} \\
& \quad-1.0535 C R^{* 3}+0.0953 Q^{* 2} * C R^{*}+0.6015 Q^{*}{ }^{*} C R^{* 2}
\end{aligned}
$$

For simplicity, the term "normalized" is omitted in the following discussion. The $R_{\text {total }}$ prediction 
Table 3. Optimal charge ratios and corresponding thermal resistances for heat loads ranging from 10 to $110 \mathrm{~W}$.

\begin{tabular}{lll}
\hline $\mathrm{Q}(\mathrm{W})$ & Predicted CR $(\%)$ & Predicted $\mathrm{R}_{\text {total }}\left({ }^{\circ} \mathrm{C} / \mathrm{W}\right)$ \\
\hline 10 & 31.1 & 2.02 \\
20 & 33.1 & 1.65 \\
30 & 35.0 & 1.33 \\
40 & 36.8 & 1.07 \\
50 & 38.4 & 0.84 \\
60 & 39.8 & 0.66 \\
70 & 41.0 & 0.52 \\
80 & 42.0 & 0.40 \\
90 & 42.9 & 0.31 \\
100 & 43.7 & 0.23 \\
110 & 44.2 & 0.17 \\
\hline
\end{tabular}

model was developed for evaluating the performance of the MLHP. This model provides crucial insights for MLHP design. The response surfaces of $R_{\text {total }}$ (Fig. 6) indicate the effects of design parameters on MLHP performance. Extreme values of the charge ratio can be derived from the partial derivative of Eq. (23) for various heating conditions. For example, the most suitable charge ratio for the lowest heat load of $10 \mathrm{~W}$ is $31.1 \%$. Moreover, the most suitable charge ratio for the highest heat load of $110 \mathrm{~W}$ is $44.2 \%$. Table 3 lists the optimal and worst charge ratios and corresponding thermal resistances for heat loads ranging from 10 to $110 \mathrm{~W}$. For these heat loads, the optimal charge ratios for the MLHP ranged from $31.1 \%$ to $44.2 \%$ and the worst charge ratios for the MLHP ranged from $71 \%$ to $84 \%$.

\section{Conclusion}

The RSM based on DOE is an efficient method for correlating the design parameters of an MLHP with its temperature response in the form of nonlinear polynomials. The derived polynomials can indicate the influence of each design parameter on the thermal response. RSM modeling considers the relationships between design parameters and an output response; thus, this method can be used to improve chaotic systems, such as MLHPs, without excessive theoretical analysis.

In the developed temperature models, the temperatures in the lower part of the MLHP had to be fitted with quadratic polynomials, whereas the temperatures in the upper part of the MLHP had to be fitted with cubic polynomials. This result indirectly confirmed the variations in the thermal instability of MLHP flow. By analyzing the temperature sensitivity, the heat transfer characteristics of the MLHP were determined for various conditions. The optimal $R_{\text {total }}$ value of the MLHP ranged from 0.17 to $2.02{ }^{\circ} \mathrm{C} / \mathrm{W}$ under different heating conditions, and the corresponding charge ratio ranged from $31.1 \%$ to $44.2 \%$.
The results obtained with the proposed models were consistent with the experimental results, which proves that RSM modeling can be used to develop conjugate heat transfer systems with optimal design constraints.

\section{Conflict of interest}

The author herein declares no conflicts of interest that could prevent the publication of this article.

\section{Acknowledgments}

This research is self-studied without financial support. The author sincerely thanks the reviewers for their thoughtful and thorough reviews.

\section{References}

[1] Adachi T, Fujita K, Nagai H. Numerical study of temperature oscillation in loop heat pipe. Appl Therm Eng 2019;163: 114281.

[2] Ahmad H, Kim SK, Jung SY. Analysis of thermally driven flow behaviors for two-turn closed-loop pulsating heat pipe in ambient conditions: an experimental approach. Int J Heat Mass Tran 2020;150:119245.

[3] Akachi H. Structure of a heat pipe. U.S. Patent No. 4921041. 1990. https://patentimages.storage.googleapis.com/db/4d/ 08/b2e8835cf995b9/US4921041.pdf.

[4] Babayan AE, Margaryan NL, Nerkararyan Kh V. About the nature of increase of the nonlinear optical response of a rough surface. Photon Nanostr Fund Appl 2006;4:35-40.

[5] Danmaliki GI, Saleh TA, Shamsuddeen AA. Response surface methodology optimization of adsorptive desulfurization on nickel/activated carbon. Chem Eng J 2017;313:993-1003.

[6] Delpech B, Axcell B, Jouhara H. Experimental investigation of a radiative heat pipe for waste heat recovery in a ceramics kiln. Energy 2019;170:636-51.

[7] Gadekar MR, Ahammed MM. Modeling dye removal by adsorption onto water treatment residuals using combined response surface methodology-artificial neural network approach. J Environ Manag 2019;231:241-8.

[8] Hong S, Wang S, Zhang L. Effect of groove configuration on two-phase flow instability for ultra-thin looped heat pipes in thermal management system. Int J Therm Sci 2017;121:369-80.

[9] Hosoda M, Nishio S, Shirakashi R. Study of meandering closed-loop heat transport device (vapor-plug propagation phenomena). JSME Int J Ser B 1999;42:737-44.

[10] Khandekar S, Charoensawan P, Groll M, Terdtoon P. Closed loop pulsating heat pipes Part B: visualization and semiempirical modeling. Appl Therm Eng 2003;23:2021-33.

[11] Li J, Hong F, Xie R, Cheng P. Pore scale simulation of evaporation in a porous wick of a loop heat pipe flat evaporator using Lattice Boltzmann method. Int Commun Heat Mass Tran 2019;102:22-33.

[12] Mäkelä M. Experimental design and response surface methodology in energy applications: a tutorial review. Energy Convers Manag 2017;151:630-40.

[13] Miura M, Nagasaki T, Ito Y. Experimental investigation of heat transport with oscillating liquid column in pulsating heat pipe using forced oscillation system. Int J Heat Mass Tran 2017;106:997-1004.

[14] Nishikawara M, Otani K, Ueda Y, Yanada H. Liquid-vapor phase behavior and operating characteristics of the capillary evaporator of a loop heat pipe at start-up. Int J Therm Sci 2018;129:426-33. 
[15] Qian N, Fu Y, Zhang Y, Chen J, Xu J. Experimental investigation of thermal performance of the oscillating heat pipe for the grinding wheel. Int J Heat Mass Tran 2019;136:911-23.

[16] Singh R, Mochizuki M, Yamada T, Nguyen T. Cooling of LED headlamp in automotive by heat pipes. Appl Therm Eng 2020;166:114733.

[17] Stevens KA, Smith SM, Taft BS. Variation in oscillating heat pipe performance. Appl Therm Eng 2019;149:987-95.

[18] Sun Q, Qu J, Li X, Yuan J. Experimental investigation of thermo-hydrodynamic behavior in a closed loop oscillating heat pipe. Exp Therm Fluid Sci 2017;82:450-8.

[19] Wang $X$, Zhang N. Numerical analysis of heat transfer in pulsating turbulent flow in a pipe. Int J Heat Mass Transf 2005;48:3957-70.

[20] Xue ZH, Qu W. Experimental and theoretical research on a ammonia pulsating heat pipe: new full visualization of flow pattern and operating mechanism study. Int J Heat Mass Tran 2017;106:149-66.
[21] Yang JF, Ohji T. Evaluation of mechanical properties of porous silicon nitride produced by partial hot-pressing. Ceram Eng Sci Proc 2001;22:243-50.

[22] Yang XH, Tan SC, He ZZ, Liu J. Finned heat pipe assisted low melting point metal PCM heat sink against extremely high power thermal shock. Energy Convers Manag 2018;160: 467-76.

[23] Yaseen ZM, Keshtegar B, Hwang HJ, Nehdi ML. Predicting reinforcing bar development length using polynomial chaos expansions. Eng Struct 2019;195:524-35.

[24] Yoon A, Kim SJ. Understanding of the thermo-hydrodynamic coupling in a micro pulsating heat pipe. Int $\mathrm{J}$ Heat Mass Tran 2018;127:1004-13.

[25] Zhang Q, Lin G, Shen X, Bai L, Wen D. Visualization study on the heat and mass transfer in the evaporator-compensation chamber of a loop heat pipe. Appl Therm Eng 2020;164: 114472 . 